\title{
Optimisation of recovery protocols for double-base smokeless powder residues analysed by total vaporisation (TV) SPME/GC-MS
}

\author{
Georgina Sauzier ${ }^{\mathrm{a}, \mathrm{b}}$, Dana Bors ${ }^{\mathrm{c}}$, Jordan Ash ${ }^{\mathrm{c}}$, John V. Goodpaster ${ }^{\mathrm{c}}$ and Simon W. Lewis ${ }^{\mathrm{a}, \mathrm{b}}$ \\ ${ }^{a}$ Department of Chemistry, Curtin University, GPO Box U1987, Perth, Western Australia 6845, Australia. \\ ${ }^{b}$ Nanochemistry Research Institute, GPO Box U1987, Perth, Western Australia 6845, Australia. \\ ${ }^{c}$ Department of Chemistry and Chemical Biology, Indiana University Purdue University Indianapolis, 402 N. Blackford \\ Street, Indianapolis, IN 46202, USA.
}

\begin{abstract}
The investigation of explosive events requires appropriate evidential protocols to recover and preserve residues from the scene. In current protocols however, there is a lack of scientifically rigorous investigation concerning their validity. In this study, a central composite design was used to determine statistically validated optimum recovery parameters for double-base smokeless powder residues on steel, analysed using total vaporisation (TV) SPME/GC-MS. It was found that maximum recovery was obtained using isopropanol-wetted swabs stored under refrigerated conditions for a minimum period, then extracted for 15 minutes into acetone. These parameters were applied to the recovery of post-blast residues deposited on steel witness surfaces following a PVC pipe bomb detonation, resulting in detection of all target components across the majority of samples. Higher overall recoveries were obtained from plates facing the sides of the device, consistent with the point of first failure occurring in the pipe body as observed in previous studies. The methodology employed here may be readily applied to a variety of other explosive compounds, and thus assist in establishing 'best practice' procedures for explosive investigations.
\end{abstract}

\section{Introduction}

In recent decades, improvised explosive devices (IEDs) have become an increasing topic of public concern, with high-profile incidents such as the Bali, London bus and Boston Marathon bombings garnering mass international attention. Although the majority of media coverage has focussed on large-scale incidents, there has also been a rise in nuisance explosions involving smaller devices such as pipe bombs. This is particularly the case in the United States, where pipe bombs are considered to make up the vast majority of IED encounters [1, 2]. These devices can be easily constructed from everyday materials, and the containment provided by the pipe can produce a large explosive effect using simple low-order explosives. 
In the US, pipe bombs frequently employ smokeless gunpowders as the explosive charge. Smokeless powders are a class of nitrocellulose-based propellants designed to produce minimal solid residue upon firing, and can be categorised as single-, double- or triple-base depending on their composition. Single-base powders rely solely on nitrocellulose as the energetic component, while double-base smokeless powder (DBSP) also contains nitroglycerin for increased detonation velocity [3-5]. Triple-base powders additionally employ nitroguanidine to lower the flame temperature and reduce barrel erosion, although these formulations are restricted to large calibre munitions and are hence rarely encountered in IEDs [6, 7]. All three varieties of smokeless powder may also contain additives, such as diphenylamine or ethyl centralite, that act as stabilisers, plasticisers, opacifiers or flash suppressants [8].

Smokeless powders are favourable due to their ready availability, with 10 million pounds produced commercially in the US each year [9]. They are also relatively powerful; decomposing at rates of up to 1,000 metres per second; and can be purchased loose at sporting goods stores in quantities of up to 25 pounds [7, 9]. Consequently, smokeless powders comprised over $20 \%$ of powder-related explosive incidents reported to the US Bomb Data Centre between 2008 and 2014 (J. Clyburne, US Bomb Data Centre, personal communication, 2015). The investigation of these events relies upon the detection and identification of residues from the scene. To this end, a large volume of research has focussed on developing highly sensitive and selective instrumental methods for the analysis of explosive materials. However, the success of these methods are largely dependent on the sampling method, storage conditions and extraction parameters employed prior to instrumental analysis [10-12]. Adequate research to develop appropriate sampling and handling procedures is thus of equally critical importance.

A common sampling method for explosive residues, particularly on large, non-porous surfaces, is the use of swabbing techniques. A number of studies have thus evaluated various swabbing materials, solvents or extraction procedures in the recovery of both organic and inorganic explosives [13-20]. The findings of these studies have been varied, with the best swabbing media and wetting or extraction solvents often differing according to the recovery surface or target analytes utilised [15-17]. Investigations have also been conducted regarding the stability of explosive residues stored under different conditions. These inquiries have established that volatile explosives such as TATP may rapidly evaporate at room temperature, while nitroaromatics such as TNT are prone to photo-transformation if left exposed to light [13, 21, 22]. Suitable storage conditions must be determined for such samples in order to preserve their evidential value. 
Despite a number of studies concerning the sampling, storage or extraction of explosive residues, there is a lack of research investigating these factors concurrently. Song-im et al. investigated a combination of swab types and solvents for the recovery of explosive residues on different substrates; however, the same extraction parameters were employed for all samples, and no analysis was made of the effect of different storage conditions [16]. Similarly, DeTata et al. examined a combination of swab types, solvents and agitation methods for the recovery of explosive residues, but utilised only a single swab type and solvent to investigate the stability of residues stored at different temperatures [13]. This univariate, one-at-a-time approach fails to take into account potential interactions between the factors, and the effect that these may have on explosives recovery. Additionally, studies to date have relied solely upon visual assessment of the acquired data. Interaction effects may not be readily evident from the raw data, and can thus be overlooked when relying on manual inspections.

Effective optimisation requires statistical methods or algorithms to identify and model interactions within the dataset. One such approach is the use of experimental design methods such as a central composite design (CCD). This is a multivariate technique that models the relationships between several explanatory factors, such as sampling or storage parameters; and a dependent response, such as the consequent explosives recovery [23, 24]. The resultant data can be used to determine the factor levels that will produce a target response, as well as determining the significance and effect of any factor interactions [25]. CCDs are particularly desirable due to their high efficiency; providing the necessary information in a minimal number of experiments [23]. Additionally, unlike many other experimental designs, CCDs are capable of modelling curvilinear variable effects [26, 27]. Recent studies have successfully utilised CCDs to optimise the extraction and instrumental analysis parameters of propellant or nitroaromatic explosive residues [28-30]. In spite of these findings, the broader capability of experimental design to investigate initial factors such as appropriate sampling and storage methods remains unexplored.

In this study, a CCD was employed to investigate sampling, storage and extraction procedures for the recovery of DBSP residues analysed using total vaporisation (TV) SPME/GC-MS. This method has been demonstrated as offering greater sensitivity than liquid injection or headspace or immersion SPME [31-33], and has been successfully applied to the detection of smokeless powder residues on steel pipe bomb fragments [34]. The optimised parameters were then applied to post-blast residues deposited on witness materials following detonation of a pipe bomb device. 


\section{Materials and Methods}

\section{Materials}

Acetone (analytical grade, Fischer Scientific) was used as received. Alliant Red Dot double-base smokeless powder was obtained from Gander Mountain, and a solution of this powder $(1.022 \mathrm{~g}$ in $500 \mathrm{~mL}$ ) prepared in acetone. PVC piping was sourced from Home Depot and low carbon galvanised steel sheets from Grainger Industrial Supply. SPME fibres were purchased from Sigma Aldrich, and SPME vials and caps from Gerstel.

\section{Optimisation design}

A central composite design was generated using Minitab 17 software (Minitab Inc., Pennsylvania, US) to investigate the choice of sampling solvent, storage location, storage duration and extraction time as shown in Table 1. A face-centered CCD $(\alpha=1)$ with 8 replicates of each centre point was selected. This design was chosen as only three rather than five levels are required for each factor, and because it allowed the number of storage days at each level to be restricted to integer values. This resulted in a total of 78 experiments. Analysis of the design was conducted using Minitab 17, optimising the recovery of nitroglycerin, diphenylamine and ethyl centralite to a maximum. Two-way ANOVA was conducted as part of the design analysis to assess the significance of any individual or two-way factor interactions.

Table 1: Factors and levels tested for face-centred central composite design.

\begin{tabular}{ll}
\hline & Levels \\
\hline Swab Type & None (dry), acetone and isopropanol \\
Storage Location & Fume cupboard $\left(\sim 25^{\circ} \mathrm{C}\right)$ and refrigerator $\left(\sim 8{ }^{\circ} \mathrm{C}\right)$ \\
Storage Duration & 0,3 and 6 days \\
Extraction Time & 15,37 and 60 minutes
\end{tabular}

\section{Sample preparation}

Low carbon galvanised steel sheets (48 in x 48 in x 0.019 in) were utilised as the recovery surface for all samples. Each sheet was cut into 16 plates (12 in x 12 in), then further divided into 3 in x 3 in squares by scoring grid-lines into one side of each plate. The surface was cleaned using deionised water followed by ethanol and acetone, and allowed to completely dry at room temperature before use. Samples for each experiment were prepared by spiking $50 \mu \mathrm{L}$ of smokeless powder solution onto squares of the galvanised steel grid, and allowing the solvent to dry prior to sampling. 


\section{Sample swabbing and extraction}

Swabbing was carried out used Puritan sterile cotton-tip applicators. For wetted swabbing, the cotton-tips were briefly immersed in the desired solvent and the excess allowed to evaporate for 20 seconds. The cotton-tips were then rubbed back and forth across the sampling area for 10 seconds, and the tips placed in $12 \mathrm{~mL}$ screw-top vials. Each vial was sealed using wax film, and placed in either a fume cupboard at room temperature or in a refrigerator for the required storage duration. For extraction, $5 \mathrm{~mL}$ of acetone was pipetted into each vial and the vial re-sealed using wax film. Vials were placed onto a WR Standard Analog Shaker Table on setting 3 for the desired extraction time. $60 \mu \mathrm{L}$ of the extract was transferred to a $20 \mathrm{~mL}$ SPME vial for analysis.

\section{Instrumental analysis}

All analyses were conducted using a Thermo Trace Ultra GC equipped with a DSQ II MS and TriPlus Autosampler. The method utilised was adapted from that developed and optimised by Bors for DBSP analysis [34] in order to provide a shorter analysis time. Samples were incubated for 1 minute at $60{ }^{\circ} \mathrm{C}$, followed by a 5 minute extraction onto a $100 \mu$ m polydimethylsiloxane (PDMS) SPME fibre. After extraction, the fibre was desorbed in the GC inlet for 1 minute in splitless mode. A PTV inlet ramp was used with an initial temperature of $200{ }^{\circ} \mathrm{C}$ for 0.25 minute, ramped $10{ }^{\circ} \mathrm{C} \mathrm{s}^{-1}$ to $250{ }^{\circ} \mathrm{C}$ and held for 1 minute. The fibre was conditioned offline at $240{ }^{\circ} \mathrm{C}$ for 5 minutes between each injection.

Separation was carried out on a Zebron ZB5-MS column (10 m x $0.18 \mathrm{~mm}$ x $0.18 \mathrm{~mm}$ ), using helium as the carrier gas with a flow rate of $1.5 \mathrm{~mL} \mathrm{~min}^{-1}$. The oven program was initiated at 40 ${ }^{\circ} \mathrm{C}$ held for $1 \mathrm{~min}$, ramped at $40{ }^{\circ} \mathrm{C} \min ^{-1}$ to $300^{\circ} \mathrm{C}$, and held for a further $1 \mathrm{~min}$. The transfer line to the MS was held at $250^{\circ} \mathrm{C}$. Pulsed positive ion negative ion chemical ionisation (PPINICI) was used with a $200{ }^{\circ} \mathrm{C}$ ion source and methane reagent gas flow of $2 \mathrm{~mL} \mathrm{~min}{ }^{-1}$. Selected ion monitoring (SIM) was used to detect nitroglycerin (m/z 42 and m/z 62 in negative mode, dwell time $25 \mathrm{~ms}$ ), diphenylamine (m/z 170 in positive mode, dwell time $50 \mathrm{~ms}$ ) and ethyl centralite (m/z 269 in positive mode, dwell time $100 \mathrm{~ms})$.

\section{Pipe bomb trial}

Assembly and detonation of the pipe bomb device was completed by the Indiana State Police Bomb Squad. A single device was constructed using PVC pipe (8 in $\mathrm{x} 1$ in diameter) and endcaps (1 in diameter), with an approximately $50 \mathrm{~g}$ charge of Alliant Red Dot powder. The device was suspended by wire within a welded metal frame, with the end-caps pointing in the north and south directions. Six galvanised steel plates (12 in x 12 in) were attached to each side of the frame 
(including above and below the device) by threading metal wire through holes drilled into the corners of each plate.

A time fuse inserted through a hole in the right endcap was used to initiate the device. Following detonation, the steel plates were cut along the previously scored lines. The resulting segments (3 in $x 3$ in) were each given a unique alphanumeric identifier, and stored in individual clip-seal bags prior to analysis. Sampling, storage and extraction were conducted using the optimal parameters determined by the central composite design.

\section{Results and Discussion}

\section{Preliminary considerations}

To date, the majority of studies concerning the optimal recovery of explosive residues have utilised standard solutions of the target analytes, rather than 'real' residues generated through deflagration or detonation. This is largely due to the difficulty in controlling these processes to obtain consistent deposition of residues [35], as well as the practicality and safety issues in conducting a large number of explosive experiments. It must be noted, though, that the use of standard solutions omits potential interferences caused by other components of the explosive material, such as plasticisers or surface modifiers. For this study, residues were thus simulated using a solution of the propellant rather than just the target analytes, in order to provide a more realistic representation of the post-blast residue composition. A detonation trial employing the propellant in an explosive device was then conducted to test the developed recovery protocols on real residues, in addition to those simulated under controlled laboratory conditions.

Samples for the optimisation study needed to be prepared and analysed across multiple days, which could potentially result in extraneous variation. Such variations can typically be managed through blocking; wherein any 'nuisance' factors; i.e. those that have an effect on the response but are not of primary interest; are held constant whilst that of interest is permitted to vary [27, 36]. In this manner, the effect of the factor of interest can be evaluated without interference of the nuisance factor. In this case, as the storage duration of the samples was a factor under investigation, it was not possible in this design to block for both the date of sample preparation and that of analysis. For practicality, it was decided to block samples according to their preparation date, with experiments in each block randomised in order to reduce any remaining nuisance effects as much as possible. Nevertheless, a degree of uncontrolled variation may still be present in the results. 


\section{Optimisation}

A face-centered central composite design was used to optimise the response of the three target components to a maximum. Although results are presented for all three components, the primary goal was to determine suitable protocols for the recovery of nitroglycerin. Under most forensic examination protocols, this component must be detected in order to report the presence of DBSP residues. Additional components such as diphenylamine and ethyl centralite may assist in identifying the brand of powder utilised, but these alone are not indicative of an explosive material.

The optimum parameters and corresponding desirability for each individual component are shown in Table 2. The desirability is a measure of how suited the parameters are to producing an optimum response, with values ranging from 0 (least desirable) to 1 (most desirable). It can be seen that nitroglycerin and diphenylamine exhibit the same optimum parameters, with excellent desirability in both instances. The parameters for ethyl centralite, on the other hand, differ from the previous components and show a much lower desirability. This is likely due to the lower detection rate of ethyl centralite (82\%), resulting from its low vapour pressure in comparison to nitroglycerin or diphenylamine (100\% detection). The overall optimum parameters, while the same as those determined for nitroglycerin and diphenylamine, thus exhibit a decreased desirability due to the poor response expected from ethyl centralite. Regardless, as the desirability towards nitroglycerin was very high, these parameters were used for the remainder of the study.

Table 2: CCD optimised parameters for the recovery of double-base smokeless powder components.

\begin{tabular}{lllll}
\hline & Nitroglycerin & Diphenylamine & Ethyl Centralite & Overall \\
\hline Swab Type & Isopropanol & Isopropanol & Dry & Isopropanol \\
Storage Location & Fridge & Fridge & Fridge & Fridge \\
Storage Duration & 0 Days & 0 Days & 6 Days & 0 Days \\
Extraction Time & 15 Minutes & 15 Minutes & 60 Minutes & 15 Minutes \\
Desirability & 1.000 & 0.996 & 0.672 & 0.777
\end{tabular}

Two-way ANOVA was used to evaluate the effect of each factor on the subsequent explosives recovery (Table 3). The choice of swabbing solvent was not identified as a critical factor for any of the three target components, possibly due to the similarity in recoveries obtained using acetone and isopropanol. The extraction time was also not found to be significant, despite a decreased 
recovery for all three components between the 15 and 37 minute extraction times (Figure 1). This is potentially due to adsorption of the residues onto the glass surface of the extraction vials during sample agitation, which has been reported in other studies [13, 21]. Nitroglycerin and diphenylamine recoveries were slightly improved upon further extraction, although the use of a minimum extraction time still yielded optimum results.

Recoveries of diphenylamine and ethyl centralite were substantially lower in samples stored at room temperature than those under refrigerated conditions $(p<0.00)$. Conversely, storage temperature was found to have low significance on the recovery of nitroglycerin ( $p=0.049)$. It was expected that losses would occur at higher temperature due to residue evaporation, and that this would be most pronounced in the recovery of nitroglycerin due to its higher volatility than diphenylamine or ethyl centralite. The results obtained here instead suggest that the decreased recoveries are more likely due to solvent evaporation from the swab material, resulting in increased binding of the residues as reported by DeTata et al. [13]. The lower decrease in nitroglycerin recovery indicates that this component exhibits a lesser degree of binding to the swab material, allowing it to be more readily extracted when the swab is re-wetted.

Both diphenylamine and ethyl centralite were significantly affected by the storage duration, with longer storage periods proving detrimental to the former $(\mathrm{p}<0.00)$ whilst appearing to improve recoveries of the latter $(p=0.003)$. This is evident from the response surface plots (Figure 2), which illustrate how recoveries of the components vary according to the continuous factors; storage and extraction time. It can be seen that the anticipated response steadily increases with storage duration for ethyl centralite, whilst a minimum storage period produces the optimum recovery for diphenylamine.

Interestingly, the response plot of both diphenylamine and nitroglycerin exhibit a response curvature associated with the storage duration of samples, in which those stored for 3 days exhibit much lower recoveries than those stored for 0 or 6 days. This curvature was found to be significant for both compounds ( $\mathrm{p}<0.005)$. Although the initial decrease in recovery could be anticipated due to residue loss or decomposition while in storage, the increased recovery between three and six days of storage was not anticipated. It should be noted that due to the analysis of samples across multiple days, this curvature may be a result of block effects as previously discussed. Further work is hence required to determine whether the results obtained here can be reproduced, or are the result of extraneous variations in the data. 


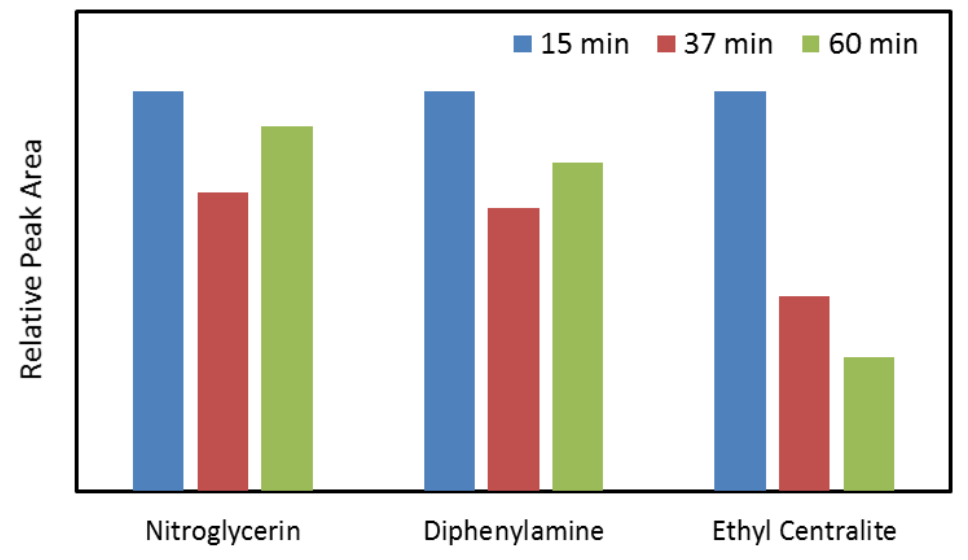

Figure 1: Relative GC-MS peak areas of nitroglycerin, diphenylamine and ethyl centralite recovered from isopropanol-wetted swabs under refrigerated conditions, using $15 \mathrm{~min}, 37 \mathrm{~min}$ and $60 \mathrm{~min}$ extraction times. Peak areas have been normalised to maximum recovery to allow comparison of all three components.

Table 3: $p$-values for all individual factors or interactions in the recovery of DBSP residues on steel. $(*)$ denotes a significant effect

Nitroglycerin Diphenylamine Ethyl centralite

\begin{tabular}{lccc}
\hline Sampling solvent & 0.999 & 0.500 & 0.541 \\
Storage location & $0.049 *$ & $0.000^{*}$ & $0.000^{*}$ \\
Storage duration & 0.292 & $0.000^{*}$ & $0.003^{*}$ \\
Extraction time & 0.627 & 0.391 & 0.327 \\
Storage duration curvature & $0.000 *$ & $0.000 *$ & $0.028 *$ \\
Extraction time curvature & 0.925 & 0.472 & 0.695 \\
Storage duration * Extraction time & 0.058 & $0.012 *$ & 0.176 \\
Storage duration * Sampling solvent & 0.964 & 0.478 & 0.408 \\
Storage duration * Storage location & 0.612 & 0.716 & 0.387 \\
Extraction time * Sampling solvent & 0.876 & 0.646 & 0.271 \\
Extraction time * Storage location & 0.838 & 0.865 & 0.788 \\
Sampling solvent * Storage location & 0.203 & 0.563 & 0.842
\end{tabular}




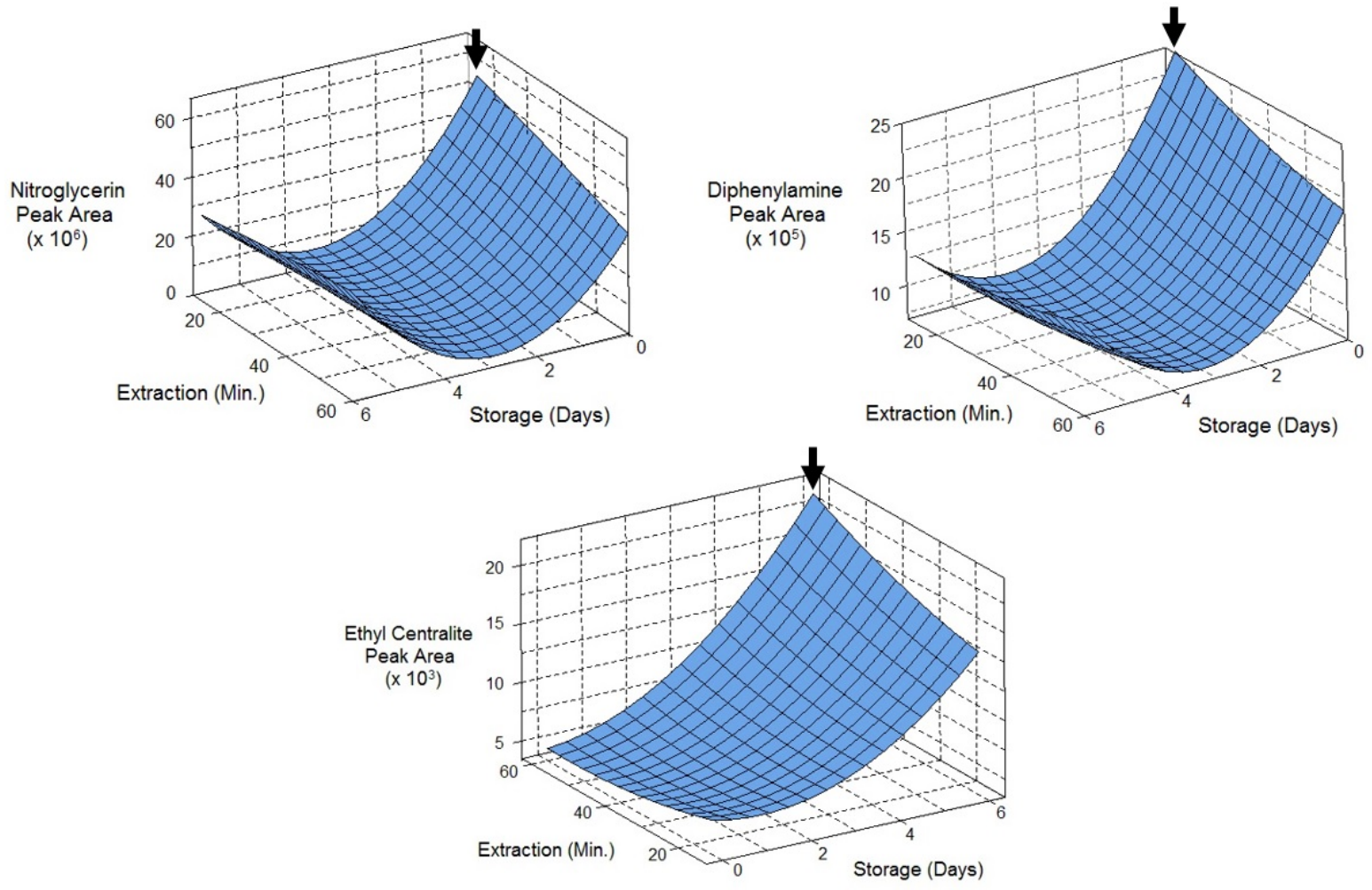

Figure 2: Response surface plots for the recovery of nitroglycerin and diphenylamine (isopropanol-wetted swabs) and ethyl centralite (dry swabs) stored under refrigerated conditions, as a function of extraction and storage time. Arrows indicate optimum points on each response surface.

Interactions between the different factors were generally found to be insignificant, with exception of the storage and extraction times. The combination of these factors was found to be influential on the recoveries of diphenylamine $(p=0.012)$ and ethyl centralite $(p=0.028)$, with longer extractions required for samples stored over 3 or 6 days than those analysed on the same day as preparation. These findings are again consistent with increased residue binding onto the swab material whilst in storage, resulting a longer period to extract the residues for analysis. This interaction demonstrates the value of a central composite design, as univariate optimisation of the extraction time following three or six days of storage could produce a false optimum extraction time of 60 minutes. By employing a CCD to model the entire response surface, it can be seen that the true optimum is in fact obtained when both the storage and extraction time are minimised.

\section{Pipe bomb trial}

As mentioned previously, the optimisation design was carried out using dissolved DBSP as a simulated residue matrix, in order to obtain reproducible deposits for sampling. However, actual residues generated through an explosive event are significantly more complex due to the changes that occur in the explosive as it reacts, degradation processes following reaction, and potential 
contamination from the local environment [37, 38]. The optimised parameters were therefore tested on post-blast residues generated through a PVC pipe bomb detonation, in order to evaluate their applicability within an operational context.

Previous work by Bors et al. employed TV-SPME/GC-MS for the recovery of DBSP residues from steel pipe bomb device fragments [34]. Although the device itself is a key source of postblast residues, traces may also be recovered from "witness” materials surrounding the explosive charge [38]. This trial therefore aimed to recover residues on surfaces proximate to the device, rather than the device itself. Galvanised steel was chosen as the witness surface as it is a common non-porous material that could be expected to survive an explosive blast, providing an ideal target substrate for explosives sampling through sampling techniques. Pre-cut steel plates were attached to each side of a metal frame surrounding the device, which was suspended using in the centre of the frame using metal wire. Following detonation of the device (Figure 3), each plate was divided into 16 areas for sampling using the CCD-selected parameters. This resulted in excellent recovery rates of nitroglycerin (99.0 \%) and diphenylamine (95.8 \%), and moderate recovery of ethyl centralite (64.6\%).

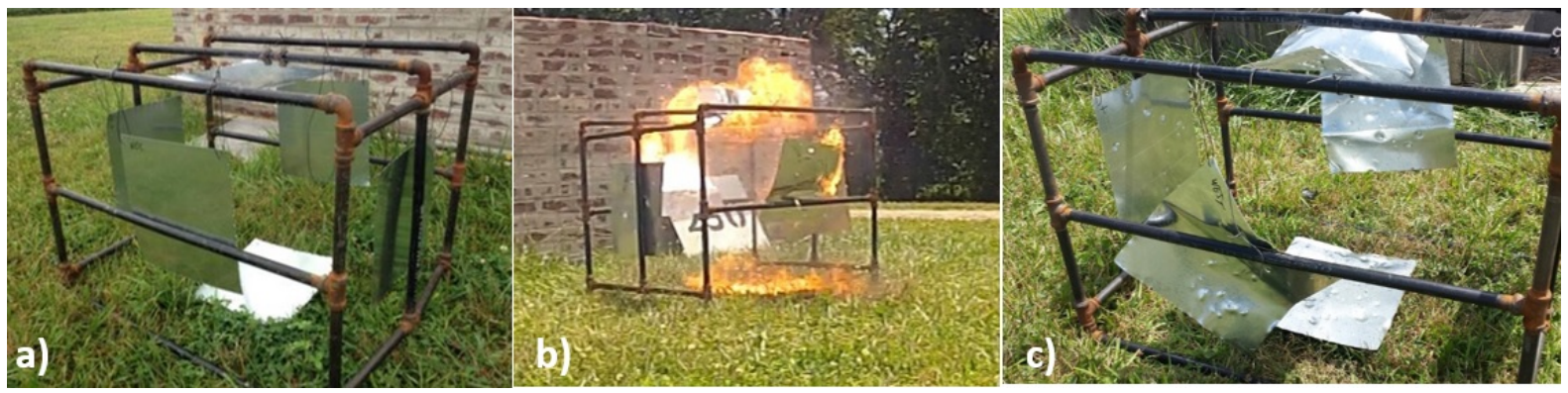

Figure 3: Photographs of the PVC pipe bomb trial set-up, a) prior to; b) during; and c) following initiation of the device.

Due to time limitations associated with this study, quantitation of the components could not be performed. The GC-MS peak areas are thus provided as a representation of explosives recovery. Average relative peak area recoveries from each witness plate are provided in Table 4. It can be seen that the highest overall recoveries were obtained from plates facing the sides of the device (east and west), rather than the end-caps. This is consistent with previous observations that the initial containment failure of PVC pipe bomb devices initiates in the pipe body, with the explosive plume primarily expanding from the sides of the device [39]. It should be noted that in pipe bombs constructed from steel, the first breach of the container has instead observed to occur 
at the end-caps, which would presumably result in a differing distribution of post-blast residue on surrounding surfaces.

Table 4: Relative GC-MS peak areas (normalised to maximum) of nitroglycerin, diphenylamine and ethyl centralite from galvanised steel witness plates following detonation of a PVC pipe bomb device. Values are averaged across 16 samples.

\begin{tabular}{llll}
\hline Plate Position & Nitroglycerin & Diphenylamine & Ethyl Centralite \\
\hline East & 1.00 & 1.00 & 1.00 \\
West & 0.95 & 0.68 & 0.51 \\
North & 0.00 & 0.01 & 0.00 \\
South & 0.34 & 0.21 & 0.14 \\
Top & 0.54 & 0.33 & 0.78 \\
Bottom & 0.47 & 0.38 & 0.87
\end{tabular}

\section{Conclusions}

The use of a CCD in combination with TV-SPME GC/MS analysis was successfully used to develop statistically valid optimised protocols for the sampling, storage and extraction of doublebase smokeless powder residues. Storage temperature was identified as the most significant single factor, with recoveries of all three target components decreasing substantially when samples were not stored at low temperature. Varying recoveries were obtained with different swabbing solvents and extraction times, with isopropanol-wetted swabs extracted for 15 minutes giving the best results. However, neither of these factors alone were determined as being statistically significant. Minimum storage durations were generally found to be ideal, with a substantial curvature in response also observed across the 0-6 day period tested, although this is potentially the result of block effects in the experimental design. As expected, longer storage durations also resulted in a longer extraction time required to desorb residues from the swabbing material. The presence of both curvature and factor correlations in the response surface also highlight the necessity of multivariate optimisation methods to detect and model higher-order interactions within complex datasets.

Application of the optimised parameters to real residues produced from a pipe bomb detonation resulted in successful recovery of all three target components, with nitroglycerin and diphenylamine each detected in over $95 \%$ of samples. The distribution of post-blast residues about the device was found to be higher on witness materials facing the sides of the device, 
consistent with the initial failure of PVC devices occurring in the pipe body. Future work may investigate the distribution of residues following detonation of steel-constructed devices, which have instead been observed to fracture at the end-caps.

It should be noted that although smokeless powder is widely used in the US, it is rarely encountered in countries such as Australia due to legal restrictions. In fact, of 41 powder-related explosive incidents reported to the Australian Federal Police between 2008 and January 2014, only seven were confirmed to involve smokeless formulations (G. Day, Australian Federal Police, personal communication, 2014). In these jurisdictions, explosive incidents are more likely to involve mining munitions or homemade peroxide explosives, such as TNT or TATP. Future work is thus required determine optimum protocols for a wider range of explosives encountered in different geographical contexts.

The methodology presented in this study may also be applied to alternative forms of physical evidence. As with explosives analysis, the use of experimental design within a broader forensic context has been largely limited to the optimisation of instrumental analysis, rather than preanalysis procedure such as sample collection or storage. The use of multivariate optimisation methods will allow the development of scientifically rigorous evidential procedures across multiple forensic disciplines.

\section{Acknowledgements}

The authors wish to acknowledge the members of the Indiana State Police Bomb Squad and Indianapolis Ordnance (Connersville, IN) for facilitating the pipe bomb detonation trial, as well as the students at IUPUI who assisted in the preparation of witness plates or collection of postblast samples. Jasmina Clyburne (US Bomb Data Centre) and Geoff Day (Australian Federal Police) are thanked for providing statistics concerning reported explosives incidents. GS acknowledges the support of the Royal Australian Chemical Institute in the form of a Postgraduate Student Travel Bursary in order to travel to the US. GS is supported by an Australian Postgraduate Award. 


\section{References}

1. Explosives, in Criminalistics: Forensic Science, Crime, and Terrorism, J.E. Girard, Editor. 2011, Jones \& Bartlett Learning: Sudbury, MA. p. 415-442.

2. Bender, E.C. and A.D. Beveridge, Investigation of Pipe Bombs, in Forensic Investigation of Explosions, A. Beveridge, Editor. 2011, CRC Press: Boca Raton, Florida. p. 429-492.

3. Energetic Materials and Explosive Devices, in The Counterterrorism Handbook, F. Bolz, K.J. Dudonis, and D.P. Schulz, Editors. 2011, CRC Press: Boca Raton, FL. p. 241-260.

4. Heard, B.J., Firearms: Propellants - History, in Wiley Encyclopedia of Forensic Science. 2009, John Wiley \& Sons: New Jersey.

5. Bell, S., Encyclopedia of Forensic Science. 2008, New York: Infobase Publishing.

6. Hopler, R.B., The History, Development, and Characteristics of Explosives and Propellants, in Forensic Investigation of Explosions. 2011, CRC Press: Boca Raton, Florida. p. 1-18.

7. National Research Council - Committee on Smokeless and Black Powder, Black and Smokeless Powders: Technologies for Finding Bombs and the Bomb Makers. 1998, Washington, DC: National Academies Press.

8. Explosions and Explosives, in Forensic Science: An Encyclopedia of History, Methods and Techniques, W.J. Tilstone, Editor. 2006, ABC-CLIO: Santa Barbara, California.

9. Heramb, R.M. and B.R. McCord, The manufacture of smokeless powders and their forensic analysis: A brief review. Forensic Science Communications, 2002. 4(2).

10. Saferstein, R., Criminalistics: An Introduction to Forensic Science. International ed. 2011, Upper Saddle River, NJ: Pearson Prentice Hall.

11. Tamiri, T. and S. Zitrin, Explosives: Analysis, in Encyclopedia of Forensic Sciences, J.A. Siegel, P.J. Saukko, and M.M. Houck, Editors. 2013, Academic Press: Waltham. p. 64-84.

12. Todd, C., L. Jones, and M. Marshall, Explosions, in Crime Scene to Court: The Essentials of Forensic Science, P. White, Editor. 2010, RSC Publishing: Cambridge.

13. DeTata, D.A., P.A. Collins, and A.J. McKinley, A comparison of common swabbing materials for the recovery of organic and inorganic explosive residues. Journal of Forensic Sciences, 2013. 58(3): p. 757-763.

14. Perret, D., et al., LC-MS-MS determination of stabilizers and explosives residues in handswabs. Chromatographia, 2008. 68(7-8): p. 517-524.

15. Song-im, N., Explosive residue analysis: evaluation and optimisation of sampling, storage and cleanup protocols. 2011, University of Canberra.

16. Song-im, N., S. Benson, and C. Lennard, Evaluation of different sampling media for their potential use as a combined swab for the collection of both organic and inorganic explosive residues. Forensic Science International, 2012. 222(1-3): p. 102-110.

17. Szomborg, K., et al., Residues from low-order energetic materials: The comparative performance of a range of sampling approaches prior to analysis by ion chromatography. Forensic Science International, 2013. 233(1-3): p. 55-62.

18. Twibell, J.D., Assessment of solvents for the recovery of nitroglycerine from hands using cotton swabs. Journal of Forensic Sciences, 1982. 27(4): p. 792-800.

19. Thompson, R.Q., et al., Aqueous recovery from cotton swabs of organic explosives residue followed by solid phase extraction. Journal of Forensic Sciences, 1999. 44(4): p. 795-804.

20. DeTata, D.A., P.A. Collins, and A.J. McKinley, A comparison of solvent extract cleanup procedures in the analysis of organic explosives. Journal of Forensic Sciences, 2013. 58(2): p. 500-507.

21. Song-im, N., S. Benson, and C. Lennard, Stability of explosive residues in methanol/water extracts, on alcohol wipes and on a glass surface. Forensic Science International, 2013. 226(1-3): p. 244-253.

22. Kolla, P., Stability of explosives traces on different supports. Forensic Science International, 1993. 60: p. 127-137.

23. Khuri, A.I. and S. Mukhopadhyay, Response surface methodology. Wiley Interdisciplinary Reviews: Computational Statistics, 2010. 2(2): p. 128-149. 
24. Myers, R.H., C.M. Anderson-Cook, and D.C. Montgomery, Response Surface Methodology: Process and Product Optimization Using Designed Experiments. 3rd ed. ed. 2014, Somerset, NJ, USA: Wiley.

25. Bezerra, M.A., et al., Response surface methodology (RSM) as a tool for optimization in analytical chemistry. Talanta, 2008. 76(5): p. 965-977.

26. Anderson, M.J. and P.J. Whitcomb, Response Surface Methods for Optimization, in DOE Simplified: Practical Tools for Effective Experimentation. 2000, Productivity Press: New York. p. 135-145.

27. Anderson, M.J. and P.J. Whitcomb, RSM Simplified: Optimising Processes Using Response Surface Methods for Design of Experiments. 2005, Boca Raton, Florida: CRC Press.

28. Batlle, R., et al., Enhanced detection of nitroaromatic explosive vapors combining solidphase extraction-air sampling, supercritical fluid extraction, and large-volume injection-GC. Analytical Chemistry, 2003. 75(13): p. 3137-3144.

29. Fryš, O., et al., Optimization of focused ultrasonic extraction of propellant components determined by gas chromatography/mass spectrometry. Talanta, 2012. 99: p. 316-322.

30. Vanini, G., et al., Multivariate optimisation of ICP OES instrumental parameters for $\mathrm{Pb} / \mathrm{Ba} / \mathrm{Sb}$ measurement in gunshot residues. Microchemical Journal, 2015. 120: p. 58-63.

31. Bors, D. and J.V. Goodpaster. Quantitative mapping of post-blast nitroglycerin residues on pipe bomb fragments using total vaporization headspace (TV-HS) solid phase microextraction-gas chromatography/mass spectrometry (SPME-GC/MS). in American Academy of Forensic Sciences 66th Annual Scientific Meeting. 2014. Seattle, Washington: American Chemical Society.

32. Bors, D. and J.V. Goodpaster. Mapping of explosive residues on pipe bomb container fragments by a novel total vaporization technique. in ANZFSS 22nd International Symposium on the Forensic Sciences. 2014. Adelaide.

33. Rainey, C.L., D.E. Bors, and J.V. Goodpaster, Design and optimization of a total vaporization technique coupled to solid phase microextraction (TV-SPME). Analytical Chemistry, 2014. 86(22): p. 11319-11325.

34. Bors, D. and J. Goodpaster, Mapping explosive residues on galvanized pipe bomb fragments using total vaporization solid phase microextraction (TV-SPME). Analytical Methods, 2015. 7: p. 9756-9762.

35. Borusiewicz, R., G. Zadora, and J. Zieba-Palus, Chemical analysis of post explosion samples obtained as a result of model field experiments. Talanta, 2013. 116: p. 630-636.

36. Otto, M., Chemometrics: Statistics and computer application in analytical chemistry. 2nd ed. 2007, Weinheim, Germany: Wiley-VCH.

37. Marshall, M., Post-Blast Detection Issues, in Aspects of Explosives Detection, M. Marshall and J.C. Oxley, Editors. 2009, Elsevier: Boston.

38. Strobel, R.A., Recovery of Material from the Scene of an Explosion, in Forensic Investigation of Explosions, A. Beveridge, Editor. 2011, CRC Press: Boca Raton, Florida. p. 119-158.

39. Bors, D., J. Cummins, and J. Goodpaster, The anatomy of a pipe bomb explosion: The effect of explosive filler, container material and ambient temperature on device fragmentation. Forensic Science International, 2014. 234: p. 95-102. 